\title{
EDITORIAL
}

\section{Proceso de renovación y estandarización de instrucciones para la publicación en la Revista Terapéutica}

Process of renewal and standardization of instructions for publication in the Therapeutic Journal

Filiación:

1. Médico. Pediatra MSc. en Epidemiología, Dirección Instituto de Investigación y Desarrollo Científico Académico Santa Paula, Universidad Santa Paula, San José, Costa Rica.

Correspondencia: $\square$ Adriana Benavides Lara abenavides@inciensa.sa.cr

Forma de citar: Benavides Lara

A. Proceso de renovación y estandarización de instrucciones para la publicación en la Revista Terapéutica. Rev Ter. 2018;12(1):4-5

Financiamiento:

Autofinanciado

Conflictos de Interés:

El autor declara no tener conflictos de interés en la presente publicación
Adriana Benavides Lara ${ }^{1} \square$

La investigación científica es fundamental para el desarrollo de un país. Un indicador clave para cuantificar la investigación científica es la publicación de artículos en revistas indizadas, principalmente en Scopus y Science Citation Index Expanded de Web of Science.

En los últimos 20 años (1996-2016) aparecen 10248 documentos registrados en SCOPUS con al menos un autor declarado con afiliación institucional costarricense, de ellos sólo $29(0,28 \%)$ se publicaron en revistas de terapia física, terapia deportiva y rehabilitación.

En este periodo, Costa Rica se ha posicionado como el 8vo país con mayor producción en Terapia física, terapia respiratoria y rehabilitación, siendo superado por Brazil, Chile, México, Colombia, Argentina, Puerto Rico y Cuba. Costa Rica publica alrededor del $0,6 \%$ de la producción brasileña, $24,2 \%$ de la producción argentina y $61,7 \%$ de la producción cubana. Este escenario podría mejorar si se desarrollaran políticas que incentiven la publicación de artículos en revistas indizadas, lo cual podría reposicionar al país.

En Scopus existen 160 revistas a nivel mundial clasificadas como "Physical Therapy, Sports Therapy and Rehabilitation", de ellas 3 son revistas iberoamericanas, 3 brasileñas y 5 españolas. En SciELO, de 444 revistas del área biomédica, existen $2(0,45 \%)$ revistas de terapia física: fioterapia e pesquisa y fisioterapia em movimiento, ambas brasileñas.

La Revista Terapéutica de la Universidad Santa Paula se encuentra actualmente inmersa en un proceso de renovación y estandarización de criterios y normas para la publicación de artículos, lo cual le permitirá en un futuro cercano ingresar a Latindex, SciELO Costa Rica, y posteriormente postular a SCOPUS. Este proceso de renovación y estandarización ha implicado la adopción de normas y criterios estandarizados a nivel internacional en las revistas biomédicas, tanto en la estructura de cada una de las secciones de publicación con la 
respectiva adaptación de las instrucciones para los autores y adherencia a consensos internacionales de presentación deartículos siguiendo recomendaciones para cada tipo de diseño como STROBE(1) para estudios observacionales, STARD(2) para pruebas diagnósticas, CONSORT(3) para ensayos clínicos, PRISMA(4) para revisiones sistemáticas, SRQR(5) para estudios cualitativos, y $\operatorname{CARE}(6)$ para los reportes de caso. Así también, hemos adoptado la plataforma Open Journal System (OJS) como sistema único de envío, revisión, seguimiento y publicación de artículos.

Además, hemos asumido la implementación de estrategias en la promoción de la publicación científica en los diferentes estamentos de la universidad, iniciando por el nivel docente y administrativo de la universidad con capacitaciones orientadas a la búsqueda y gestión de la información científica, análisis de datos y publicación, y un acompañamiento o mentoring que nos permitirá fortalecer las competencias del recurso humano que integra la Universidad y conducir las investigaciones a una publicación científica.

\section{Referencias Bibliográficas}

1. von Elm E, Altman DG, Egger M, Pocock SJ, Gøtzsche PC, Vandenbroucke JP. Strengthening the reporting of observational studies in epidemiology (STROBE) statement: guidelines for reporting observational studies. BMJ. 20 de octubre de 2007;335(7624):806-8.

2. Bossuyt PM, Reitsma JB, Bruns DE, Gatsonis CA, Glasziou PP, Irwig L, et al. STARD 2015: an updated list of essential items for reporting diagnostic accuracy studies. The BMJ [Internet]. 28 de octubre de 2015 [cita- do 15 de abril de 2018];351. Disponible en: https://www.ncbi.nlm.nih.gov/pmc/articles/ PMC4623764/

3. Schulz KF, Altman DG, Moher D. CONSORT 2010 Statement: updated guidelines for reporting parallel group randomised trials. Trials. 24 de marzo de 2010;11:32.

4. Moher D, Liberati A, Tetzlaff J, Altman DG. Preferred Reporting Items for Systematic Reviews and Meta-Analyses: The PRISMA Statement. J Clin Epidemiol. 1 de octubre de 2009;62(10):1006-12.

5. O'Brien BC, Harris IB, Beckman TJ, Reed DA, Cook DA. Standards for reporting qualitative research: a synthesis of recommendations. Acad Med J Assoc Am Med Coll. septiembre de 2014;89(9):1245-51.

6. Gagnier JJ, Kienle G, Altman DG, Moher D, Sox H, Riley D. The CARE guidelines: consensus-based clinical case reporting guideline development. J Med Case Reports. 10 de septiembre de 2013;7:223. 